great as $R$. The method may be applied to $\kappa R$, and we have the following theorem :

Theorem : If no denominator of odd rank, after the first, in the complete quotients obtained by expanding $\sqrt{\kappa R}$ in a continued fraction, turns out to be a perfect square, the expansion being carried out until the numerator of the last convergent is greater than $\kappa R$, then the factors of $\kappa R$ differ by more than $2 \sqrt[4]{\kappa R}$.

As an example of the application of this theorem to the discovery of prime factors, take Jevons's * number $8616460799=R$. No perfect square appears in the denominators of the complete quotients obtained in expanding $\sqrt{R}$, whence one infers that the factors differ by more than $608=2 \sqrt[4]{R}$. Similar failure attends the expansion of $\sqrt{2 R}, \sqrt{6 R}$, and $\sqrt{30 R}$. On expanding $\sqrt{210 R}$, however, the third denominator is found to be the square $11881=109^{2}$. The numerator of the second convergent is 2690321 ; we know then that the numbers $2690321 \pm$ 109 contain the desired factors of the given number. The factors are 89681 and 96079 .

The most advantageous value of $\kappa$ to take is the product of the smallest distinct primes. Thus if $\kappa=30$ and the factors of $R$ are $p$ and $q$, then the factors will be discovered if $p-30 q$, $2 p-15 q, 3 p-10 q$, or $5 p-6 q$ are less than $2 \sqrt[4]{30 R}$.

Berkeley, Cat.

\title{
PROJECTIONS OF THE GLOBE APPROPRIATE FOR LABORATORY METHODS OF STUDYING THE GENERAL CIRCULATION OF THE ATMOSPHERE.
}

BY PROFESSOR CLEVELAND ABBE.

THE general circulation of the atmosphere is controlled by the general distribution of land and water, and by the insolation, with its resultant temperature, evaporation and clouds. In the analytic treatment of this problem, beginning with D'Alembert, Ferrel, and Erman, as well as in the more elegant works

* Jevons, Principles of science, p. 123, "Can the reader say what two numbers multiplied together will produce the number 8616460799 ? I think it unlikely that anyone but myself will ever know." I think that the number has been resolved before, but I do not know by whom. 
of Helmholtz, Oberbeck and Margules, it has always been considered necessary to simplify the problem by assuming a uniform surface and uniform coefficient of resistance for the whole globe, as also a uniform condition of dry air without the complexity produced by the presence of clouds. In this shape the problem is already too difficult for pure analysis, and writers have generally achieved the special solutions only by introducing numerical values based upon observations of temperature, pressure, and winds that represent existing conditions upon the earth's surface. It is likely that the profound meteorological problem, namely the deduction of the actual winds that necessarily result from the laws of mechanics, as applied to the earth, will for a long time be too difficult for pure analysis ; but the solution of this problem is greatly to be desired, and on many occasions I have stated my belief that it may be arrived at in an experimental way provided we have a laboratory properly equipped for meteorological investigations. We cannot reproduce atmospheric conditions surrounding a small globe in the laboratory, because in the free atmosphere the force of gravity is directed normal to the earth's surface, whereas in the laboratory it would be inclined to a globe at every possible angle. We must, therefore, represent any portion of the globe, with its atmosphere, by a horizontal plane surface covered with some heavy gas or liquid. The most natural thought is to adopt polar projections of the northern and southern hemispheres respectively, bounded at or beyond their equators by flexible rims that shall represent the assumed irregular variable equatorial boundary between the northern and southern circulations. This will do for those months of the year during which we may assume that the northern and southern systems do not intrude upon each other to any great extent; but of course this will fail entirely when the great Asiatic monsoon is in progress and for that season a very different laboratory experiment must be devised. We therefore for the present confine ourselves to the months October to March. Our projections of the two hemispheres, being covered with a thin layer of carbonic acid gas (or for that matter any liquid such as alcohol or water can be used as well), are to be set in steady rotation, and are to be warmed from below in such a way as approximately to imitate the actual isotherms of the lower atmosphere for any moment or day. Not only the general distribution of temperature in latitude, but the special areas of high and low temperatures, can easily be imitated by elec- 
trical resistance coils. In order that the thin layer which imitates the atmosphere shall be free from the action of the surrounding air of the laboratory our rotating planospheres are to be covered by plates of glass rotating with them, so that the upper layer of air in the model shall, like the upper layer in the atmosphere, rotate with the surrounding ether.

We have thus imagined a construction that has a certain similarity but many points of dissimilarity to the atmosphere of the earth. Our problem is to reduce these latter to a minimum, and then so to interpret the observed motions in our model that we may infer something as to the motions in the earth's atmosphere. To do this our first step must be to consider the ideas laid down by Helmholtz as to the "mechanical similarity" of the atmosphere of the earth and the model atmosphere. Various theorems relative to this similarity were first published by Helmholtz in 1873, "On a theorem relative to movements that are geometrically similar in fluid bodies," and these ideas were further applied by him in later memoirs on atmospheric motions, in 1888 and 1889 . He shows how the movements in a small model may be made geometrically and mechanically similar to those of the earth's atmosphere. The equations of motion for the model atmosphere become analogous to those of the free atmosphere by introducing certain conditions represented by three constant factors, $i . e ., \mathrm{I}$, the viscosity of the model or liquid is to be a certain multiple $q$ of the viscosity of the atmosphere; II, the density in the model is to be a certain multiple $r$ of the density of the atmosphere; III, the velocities along three coordinate axes in the model are to be equal to those in the atmosphere multiplied by a certain constant factor $n$. Under these conditions the pressures in the model will be $n^{2} r$ times the pressures in the atmosphere. The linear distances in the model will be $q / n$ times the linear distances in the atmosphere, and the time argument in the model will be $q / n^{2}$ times the time element in the free air. It is assumed that the relative movements are not so violent as to depart greatly from what is known as laminar or lamellar motion, and that the viscosity may be combined with the resistances due to convection and to the formation of discontinuous motions. Numerous applications of these principles are given by Helmholtz, and some further developments have lately been added by Lord Rayleigh.

But in applying these ideas to our two polar projections we 
stumble at once upon a great difficulty, namely that the maps are not true representations of the spherical surface of the earth. Every projection on a plane must distort the geometric relations between the points upon the original spherical surface, and students must always choose a style of projection that suits the problem they have on hand. In the present case (1) we should like to preserve the equality of surface areas, since that is proportional to the mass of the air above it ; (2) we should like to preserve equality of distances, since we have to measure and compare velocities ; but, above all, from a mechanical point of view, (3) we should like to preserve the equality of the moments of inertia, that is to say the moment of inertia of a unit mass relative to the earth's axis should be a constant fraction of the moment of inertia of the corresponding unit mass in the model with reference to the pole about which it is rotating. These three desiderata would respectively necessitate the use of (1) an equal surface development ; (2) a polyconic development; and (3) an orthographic projection. There are still other conditions with projections that correspond thereto. That which best satisfies all desiderata with the minimum sum total of errors seems to be Airy's "projection by balance of errors," published by him in 1861 as suggested by the projection used by Sir Henry James, in which latter the point of view was at the distance from the surface of the sphere equal to the radius. James's idea was to obtain a projection that should include a very large part of the surface of the earth at one view; and the map that he produced did in fact include a radius of $120^{\circ} 0^{\prime}$; this was a projection, properly so called. Airy, however, solved the problem for a development, properly so called, which should respond to the condition that the square of the distortion in distance plus the square of the distortion in area should be a minimum when one considers the sum total of the whole surface. This minimum would necessarily depend on the total area included in the map. A few months later R. A. Clarke showed that there was a slight error in Airy's computations, and then worked out a slight modification of the problem. Clarke deduced a projection, properly so called, allowing both the visual point and the plane of projection to be adjustable, so as to satisfy the same condition as that adopted by Airy in his development. The formulas and computations by Clarke's projection are far more troublesome than for Airy's development, especially when the latter are arranged in the way that $I$ have 
given. The only advantage that Clarke's projection can have is that it can be constructed graphically without the use of numerical tables or computations; but this advantage disappears as soon as we attempt to construct a large map.

The combination of Airy's development (or some projections still more appropriate) with Helmholtz's method of mechanical similarity should enable us to interpret our laboratory experiments intelligently, so that from these we may construct a close approximation to the general circulation of the atmosphere.

I consider it extremely desirable that these experiments should be made on a large scale, with due regard to all numerical, statistical and mechanical details in some laboratory where the study of meteorology is prosecuted as a branch of mathematical physics.

\section{SHORTER NOTICES.}

Breve Storia della Matematica dai tempi antichi al medio evo. By Gaetano Fazzari. Milano, R. Sandron, 1907. 268 pp. Price, 4 lire.

IT is rather strange that Italy, the country that produced the most learned bibliophile in the domain of mathematics, Prince Boncompagni, and that furnished to France another well-known collector of early works, the historian Libri, should have published so little relating to the general development of the science. It is true that Favaro and Loria have contributed very acceptably to the history of certain periods or topics, and that Riccardi's bibliography of the early mathematical works of his own country will always be a standard book of reference, but in spite of all the encouragement of men like these, and all the patriotism that would lead an Italian to write the story of a science that so largely developed on his native soil, such a work as a worthy general history of mathematics does not exist in the language of Italy. It is for this reason that such an attempt as Professor Fazzari's should be particularly welcome, the more so as it was written on the island in which Archimedes spent most of his life, which Pythagoras visited, and to which Maurolycus brought no small amount of glory in the period of the Renaissance.

The aim of Professor Fazzari has been to write a work of about the character of Ball's and Cajori's popular histories, one 